\title{
Continuation power flow considering area net interchange constraint
}

\author{
H. Avila Carhullanqui ${ }^{\mathrm{a}}$, A. Bonini Neto ${ }^{\mathrm{b}, *, 1}$, D.A. Alves ${ }^{\mathrm{a}}$ \\ ${ }^{a}$ UNESP - São Paulo State University, Department of Electrical Engineering, Ilha Solteira, SP, Brazil \\ ${ }^{\mathrm{b}}$ UNESP - São Paulo State University, Department of Biosystems Engineering, Tupã, SP, Brazil
}

\section{A R T I C L E I N F O}

\section{Article history:}

Received 18 January 2014

Received in revised form 13 January 2015

Accepted 31 January 2015

\section{Keywords:}

Continuation power flow

$P-V$ curves

Power transfer

Contingency analysis

Stead state voltage stability

Interchange limits

\begin{abstract}
A B S T R A C T
Interconnected power systems not only allow to the areas to provide mutual assistance, but also import or export energy with respect to optimize energy resources assessment where, a cost reduction involved in the generation of power required to meet its demand. To determine the required control actions, in the planning and operation stages, it is important to verify the loading margins for both the normal operation and the different conditions of contingencies that may eventually occur. In this paper a continuation power flow that allows obtaining the loading margin and maximum active power transfer considering the area interchange control is proposed. From the results of the IEEE systems ( 9 and 118 buses), a difference of up to twelve percent in the active power transfer capacity is verified compared to the cases without area interchange control. The method also highlights the effects of the loop flow which occur as a consequence of the existence of parallel paths in the interconnected network.
\end{abstract}

(c) 2015 Elsevier Ltd. All rights reserved.

\section{Introduction}

Modern electric power systems are comprised the control of the interconnected areas through tie lines (interconnecting lines). In Brazil, the system is composed predominantly by hydropower plants and some thermal power plants. Although the generation cost of hydros is lower than the thermals, they are much more far away from the consumers. Interconnected power systems allows, among other advantages, a better use of available generation resources, reduction in the required total installed capacity and generating reserve needed to ensure continuity of service, and greater operating flexibility to meet the energy demand, and consequently increasing the reliability and quality of electricity supply is obtained [1-4]. They also enable the areas to import (buy) or export (sell) power from each other. So, each area can increase/ decrease the power generation of respective generators in order to meet the increases/decreases of its own demands, or of other areas. Despite these advantages, due to the increase in demand and a greater utilization of power transfer capability of existing transmission lines, at these systems blackouts and energy rationing are still occurring [1]. Consequently, it is important to perform the assessment of the available power transfer limits of

\footnotetext{
* Corresponding author.

E-mail addresses: hilbert_1@hotmail.com (H. Avila Carhullanqui), alfredoneto@ aluno.feis.unesp (A. Bonini Neto), dalves@dee.feis.unesp.br (D.A. Alves).

${ }^{1}$ Financial support by CNPq, Brazil.
}

these interconnected systems for various energy scenarios, load conditions and network configurations, so that sufficient transmission capacity is assured. Under certain conditions the limits are restricted by voltage instability problems. In this case, it is necessary to verify that, if the system is not close to an operating condition in which a small increase in the load or in the active power transfer and an occurrence of a simple contingency causes the voltage collapse.

In studies related to static voltage stability, the continuation power flow is commonly used for tracing the system $P-V$ curves because it allows to obtain the maximum loading point (MLP) and thereby determining the accurate system loading margin, without numerical problems related to Jacobian matrix singularity [5-9]. To determine the power transfer capacity of an interconnected power system, the amount of active power to be transferred between two regions, at the same area, or areas must be defined. For that purpose, the generation in a particular region (or area), which is considered as the source region (or area), and the load on another region (or area), considered as the sink region (or area), are increased [2-4]. The net interchange of each area is defined as the algebraic sum of the active power flowing over all the tie-lines of a given area; the active power flowing to a given area is considered negative and flowing away from a given area, positive [1012]. The net interchange of each area must be kept in its respective scheduled value, which requires the addition of one equality constraint equation for each area, in solving process of the problem of load flow [10,11]. So, each area must have at least one generation bus $(P V)$ that will be used to regulate its net interchange. 
To keep the net interchange of area in its respective scheduled value, the injected active power of the regulating bus is adjusted. So, this bus is nominated by area interchange control (AIC) regulating bus and classified as $\mathrm{V}$ type, its active power generation is not specified, but only the voltage magnitude [11]. Note that if one or more areas do not have regulating bus, the net interchange cannot be controlled in this area. In a system with $\mathrm{n}$ areas only $n-1$ can be controlled because one of the net interchange is determined by specification of the rest and so, one area must be a reference area. One generator bus of the reference area is used as the reference bus and others angles of the system buses are adjusted based on this reference. It is classified as a $V \theta$ type. As the losses are not known in advance of the load flow solution, it bus is also used as slack (or swing) bus to balance the active and reactive power in the system.

The maximum active power that could be transferred between regions of the same area through the internal transmission lines, or between areas across the tie-lines, without any problems related to the voltage stability, should be assessed maintaining the correct interchange values among the areas [12-15]. Nevertheless, in [12] the maximum power interchange is calculated by the sum of active power flowing over all the tie lines, at the maximum loading point. The loading is performed by considering the system as a single area with a single slack bus, i.e., it does not include the areas' interchange constraint equations. In [11], a method for inclusion of the AIC in the conventional power flow was proposed. The method stands out, when compared to others, because it considers the generated powers at the regulating buses as state variables and allows the use of multiple AIC regulating buses per area. Based on this method, in Section 'Proposed continuation power flow' the contribution of the paper that is the inclusion of AIC in continuation power flow is considered in detail. This inclusion provides greater flexibility since now, which makes it possible to determine the available transfer capacity of different areas considering the inclusion of multiple AIC regulating buses per area. Each area acts as an independent system that controls its interchange during all the procedure of system loading. In Sections 'Loop flow, an illustrative example and Performance of the proposed CPF for the modified 118-bus IEEE test system', the proposed continuation power flow (PCPF) is applied to the IEEE systems of 9 and 118 buses. The systems were divided into several areas with the aim of highlighting the differences between the loading and power transfer margins obtained with the method with and without considering the constraints imposed by the net interchange. Two different load change scenarios are considered: (1) with a constant power factor, i.e. the active and reactive loads are increased proportionally to the base case and (2) only the active loads are increased.

Moreover, in Section 'Loop flow, an illustrative example', the results demonstrate the effects of the loop flows (parallel path flows, inadvertent flows or circulating flows), which occur due to the existence of parallel paths in the interconnected network [16-18]. As a consequence of the net interchange is the difference between the input and output power of the area, the power transfer across an affected area will not appear in it. On the order hand, as a consequence, an area that is not participating in the exchange of power has to cover the losses incurred by these unscheduled power flows and the power transfer capacity between two regions of its network will be restricted. Moreover, the AIC regulating buses will be increase or decrease their generations in order to provide the loss variations. In Section 'Performance of the proposed CPF for the modified 118bus IEEE test system', some critical contingencies of IEEE-118 considering the restrictions imposed by the AIC are also evaluated. From the results it causes a difference up to $12 \%$ in the available active power transfer capacity.

\section{Proposed continuation power flow}

The proposed continuation power flow (PCPF) is developed based on the method presented in [11], which allows the representation of multiple AIC regulating buses per area and represents that the AIC effects is put into the Jacobian matrix. In this method, the equations of generated active power of regulating buses are kept, unlike the proposed method in [10], wherein they are replaced by the net interchange equations. The PCPF is used to determine the successive solutions of a load flow by considering a given load change scenario including the interchange control. The bus voltage profiles ( $P V$ curves) are traced starting from a base case (a given initial solution) up to the maximum loading point (MLP). The singularity of the Jacobian matrix of the load flow is removed by slightly modification of the power flow equations presented in [11] and by applying a local or a geometric parameterization technique [5-7].

The general power flow equation to be solved including the interchange control, are expressed compactly as:

$\mathbf{G}\left(\boldsymbol{\theta}, \mathbf{V}, \mathbf{P}_{\mathbf{G}}, \lambda\right)=\mathbf{0}$

that can be rewritten as:

$$
\begin{aligned}
& \Delta \mathbf{P}(\boldsymbol{\theta}, \mathbf{V}, \lambda)=\mathbf{P}^{\mathbf{s p}}(\lambda)-\mathbf{P}(\boldsymbol{\theta}, \mathbf{V})=\mathbf{0} \\
& \Delta \mathbf{Q}(\boldsymbol{\theta}, \mathbf{V}, \lambda)=\mathbf{Q}^{\mathbf{s p}}(\lambda)-\mathbf{Q}(\boldsymbol{\theta}, \mathbf{V})=\mathbf{0} \\
& \Delta \mathbf{P I}(\boldsymbol{\theta}, \mathbf{V}, \lambda)=\mathbf{P I}^{\mathbf{s p}}(\lambda)-\mathbf{P I}(\boldsymbol{\theta}, \mathbf{V})=\mathbf{0} \\
& \Delta \mathbf{g}\left(\mathbf{P}_{\mathbf{G}}\right)=\mathbf{g}^{\mathbf{s p}}-\mathbf{g}\left(\mathbf{P}_{\mathbf{G}}\right)=\mathbf{0}
\end{aligned}
$$

where $\mathbf{V}$ and $\boldsymbol{\theta}$ are the vectors of voltage magnitudes and phase angles, respectively. The loading factor $(\lambda)$ is used to scale up the loading and generation level. For a given bus, $k, \mathbf{P}(\boldsymbol{\theta}, \mathbf{V})$ and $\mathbf{Q}(\boldsymbol{\theta}$, V) are given by:

$$
\begin{aligned}
& P_{k}(\boldsymbol{\theta}, \mathbf{V})=G_{k k} V_{k}^{2}+V_{k} \sum_{l \in \Omega_{k}} V_{l}\left(G_{k l} \cos \theta_{k l}+B_{k l} \operatorname{sen} \theta_{k l}\right), \quad k \in P Q, \quad P V \\
& Q_{k}(\boldsymbol{\theta}, \mathbf{V})=-B_{k k} V_{k}^{2}+V_{k} \sum_{l \in \Omega_{k}} V_{l}\left(G_{k l} \operatorname{sen} \theta_{k l}-B_{k l} \cos \theta_{k l}\right), \quad k \in P Q
\end{aligned}
$$

where $\Omega_{k}$ is the set of all buses directly connected to the bus $k$, and $G_{k l}$ and $B_{k l}$ terms represent the conductance and susceptance of $(k, l)$ element in the nodal admittance matrix $\mathbf{Y}=[\mathbf{G}]+j[\mathbf{B}]$.

For a given bus, $k, P_{k}^{\mathrm{sp}}(\lambda)=\left(P_{G k 0}+\lambda \operatorname{ger}_{\mathbf{k}} \mathrm{Ptr}_{\mathbf{0}}\right)-\left(P_{L k 0}+\lambda C_{P k} S_{k 0}\right.$ $\left.\cos \left(\varphi_{k 0}\right)\right)$ is the difference between the generated and consumed active powers for load $(P Q)$ and generation $(P V)$ buses, and $Q_{k}{ }^{\mathrm{sP}}(\lambda)=Q_{G k}-\left(Q_{L k 0}+\lambda C_{O k} S_{k 0} \operatorname{sen}\left(\varphi_{k 0}\right)\right)$ is the difference between the generated and consumed reactive powers for load buses. The subscript " 0 " is used to indicate the values of respective variables in the initial condition of operation, i.e. in the base case. The constants $C_{P k}$ and $C_{Q k}$ are used to indicate a specific network loading condition: if $C_{P k}=1$ and $C_{Q k}=1$, a loading with constant power factor is considered; if $C_{P k}=1$ and $C_{Q k}=0$, only active power variations are considered. The symbol $S_{k 0}$ is used to designate the apparent power of bus $k, \varphi_{k 0}$ is the power factor angle of bus $k$. $\operatorname{Ptr}_{0}=\sum_{k=1}^{n} C_{P k} S_{k 0} \cos \left(\varphi_{k 0}\right)$ is the power to be transferred. The increase of active power at $P Q$ buses must be supplied by the $P V$ generator buses belonging to the area that will export electric power. The symbol $P_{G k 0}$ represents the active power generated in the base case at bus $k$ and ger $_{k}$ is the participation factor of generator $k$, which is calculated from the base case solution as follows.

$\operatorname{ger}_{k}=P_{G k 0} / \sum_{j=1}^{\text {nger }} P_{G j 0}$

For a given area, nger represents the number of generators (regulating buses) that will satisfy the load increase, i.e. the generators that participate in the sharing of load increase. The increase 
of reactive power at $P Q$ load buses is provided by the generating buses. Thus, the distribution of reactive power occurs as in the case of conventional power flow, but the active power transfer is distributed among the $P V$ buses and so, $\left(P_{G k 0}+\lambda \operatorname{ger}_{\mathbf{k}} \operatorname{Ptr}_{\mathbf{0}}\right)$ is the active power generated at regulating bus $k$. The last equation in (2) is added when there is more than one AIC regulating bus per area. Considering that there are $n f$ AIC regulating buses per area, then $n f-1$ additional equations per area must be added [11]. Each equation $\left(\Delta g_{(n f-1)}\right)$, which represents the difference between the active power generation of two subsequent regulating bus, is given by

$\Delta g_{(n f-1)}=g^{s p}-g\left(P_{G(n f-1)}\right)=0-\left(P_{G(n f-1)}-\alpha_{(n f-1) n f} P_{G n f}\right)$

where $\alpha_{(n f-1) n f}=\operatorname{ger}_{(n f-1)} / \operatorname{ger}_{n f}$ is the ratio between the participation factors. When only the limits of reactive power $\left(\mathbf{Q}_{\lim }\right)$ of generators are hits, their types are switched from $V$ bus type to $Q$ bus type, i.e. they still participate as AIC regulating buses, but the generated reactive power $\left(\mathbf{Q}_{\mathbf{G}}\right)$ will be kept constant at the limit $\left(\mathbf{Q}_{\mathbf{l i m}}\right)$. When the AIC regulating bus hits its active power limit, its type is switched to type $P V$ when its specified generated active power is fixed at the corresponding limit. Nevertheless, when both the active and reactive power limits are violated then the type is switched to $P Q$. If there is no other AIC regulating bus in the area, then the area is no longer able to control its net active power interchange.

$\mathbf{P I}^{\mathbf{S P}}(\lambda)$ represents the vector of scheduled net interchange. The determination of the maximum power transfer between areas is accomplished by increasing the interchange $\left(+\lambda \operatorname{Ptr}_{\mathbf{0}}\right)$ in the generating (or source) area and decreasing it $\left(-\lambda \operatorname{Ptr}_{\mathbf{0}}\right)$ in the sink area where there is a load increase. So, when the source and sink areas are the same, its net interchange is kept at its scheduled value. Note that the net interchanges of other areas, not involved in the transactions, are also kept at their scheduled values. The net interchange of an area $k\left(\operatorname{PI}_{k}(\boldsymbol{\theta}, \mathbf{V})\right)$ is calculated by [10]:

$P I_{k}(\boldsymbol{\theta}, \mathbf{V})=\sum_{i \in \Omega_{i}} \sum_{j \in \Omega_{j}}\left(g_{i j} V_{i}^{2}-V_{i} V_{j}\left(g_{i j} \cos \theta_{i j}+b_{i j} \operatorname{sen} \theta_{i j}\right)\right)$

where $i$ is the bus of interconnection of the area $k$ and $\Omega_{i}$ is the set of all buses belonging to the interconnections of the area $k$ with the other areas, $\Omega_{j}$ is the set of buses which are directly connected to bus $i$, but not belonging to area $k$.

Eq. (2) can be solved by conventional Newton's method specifying a gradual increase in $\lambda$, i.e. it is pre-specified. With its inclusion into Eq. (2), one more equation will be required. A parameterized equation $y-y^{e}=0$ is appended to Eq. (2) for tracing voltage profiles, where $y$ and $y^{e}$ are the selected variables of the continuation parameter and its predicted value respectively. In the local parameterization technique, the tracing of voltage profile starts with the loading factor $(y=\lambda)$. Close to the maximum power interchange, it change to the voltage magnitude that presents the largest variations $\left(y=V_{k}\right)$ and after a few points, it changes back to the loading factor.

Considering multiple AIC regulating buses per area and expanding (2), augmented by the parameterized equation, in Taylor's series up to the first order terms is obtained as (7).

$$
\left[\begin{array}{c}
\Delta \mathbf{P} \\
\boldsymbol{\Delta} \mathbf{Q} \\
\Delta \mathbf{P I} \\
\Delta \mathbf{g} \\
\Delta y
\end{array}\right]^{v}=\left[\begin{array}{llll}
\mathbf{H} & \mathbf{N} & \frac{\partial \mathbf{P}}{\partial \mathbf{P}_{\mathbf{G}}} & -\frac{\partial \mathbf{P}^{\mathbf{s p}}}{\partial \lambda} \\
\mathbf{M} & \mathbf{L} & \frac{\partial \mathbf{Q}}{\partial \mathbf{P}_{\mathbf{G}}} & -\frac{\partial \mathbf{Q}^{\mathbf{s p}}}{\partial \lambda} \\
\frac{\partial \mathbf{P I}}{\partial \theta} & \frac{\partial \mathbf{P} \mathbf{I}}{\partial \mathbf{V}} & \frac{\partial \mathbf{P I}}{\partial \mathbf{P}_{\mathbf{G}}} & -\frac{\partial \mathbf{P}^{\mathbf{s p}}}{\partial \lambda} \\
\frac{\partial \mathbf{g}}{\partial \theta} & \frac{\partial \mathbf{g}}{\partial \mathbf{V}} & \frac{\partial \mathbf{g}}{\partial \mathbf{P}_{\mathbf{G}}} & 0 \\
& e_{j} &
\end{array}\right]^{v}\left[\begin{array}{c}
\Delta \theta \\
\Delta \mathbf{V} \\
\Delta \mathbf{P}_{\mathbf{G}} \\
\Delta \lambda
\end{array}\right]^{v}
$$

where $\mathbf{H}, \mathbf{N}, \mathbf{M}$ and $\mathbf{L}$ are matrices that correspond to the derivatives of active and reactive powers with respect to voltage angles $(\boldsymbol{\theta})$ and voltage magnitudes $(\mathbf{V})$, respectively. $\Delta \mathbf{P}$ and $\Delta \mathbf{Q}$ represent the respective mismatch vectors of active and reactive power, and $\Delta \mathbf{P I}$ represent the net interchange power mismatches. $\Delta \mathbf{V}$ and $\boldsymbol{\Delta} \boldsymbol{\theta}$ are the respective vectors of voltage magnitude and phase angle corrections and $\mathbf{\Delta} \mathbf{P}_{\mathbf{G}}$ is the vector of generated active power correction at AIC regulating buses. The $\Delta \mathbf{g}$ equations must be excluded in the case of a single AIC regulating bus per area. The $\mathbf{e}_{\mathbf{j}}$ is a row vector with all elements equal to zero except the $j$ th, which equals 1 and corresponds to the chosen continuation parameter $(y)$. At each iteration $(v), \mathbf{V}, \boldsymbol{\theta}$ and $\mathbf{P}_{\mathbf{G}}$ are updated by

$$
\begin{aligned}
\boldsymbol{\theta}^{v+1} & =\boldsymbol{\theta}^{v}+\boldsymbol{\Delta} \boldsymbol{\theta}^{v} \\
\mathbf{V}^{v+1} & =\mathbf{V}^{v}+\Delta \mathbf{V}^{v} \\
\mathbf{P}_{\mathbf{G}}^{v+1} & =\mathbf{P}_{\mathbf{G}}^{v}+\Delta \mathbf{P}_{\mathbf{G}}^{v}
\end{aligned}
$$

\section{Loop flow, an illustrative example}

The active power transfers through regions of the transmission network are determined by the impedances of the branches (transmission line, transformer) existents in all possible parallel paths and the location of the loads and generations. So, as the flow of electric power in a network is divided among the branches, according to their respective impedances, there is a power flow passing through the transmission lines of other areas, forcing them to increase (or decrease) their generations of active power in order to match their respective losses. The generation increase (or decrease) will be controlled by the AIC, which will see a negative difference (or positive) between the output and input of active power of the area. So, a company not participating in the power exchange could have to pay for an interchange between external companies. Loop flow problems have been gaining attention over the past decades due to the increasing loop flow trend [2-4,1618]. It may contribute to network congestion, to limit the possibilities for power exchange among areas, to increase the active and reactive power losses and so, to impact the economic operation voltage security margin of the involved neighboring systems.

Fig. 1 shows a simple interconnected system consisting of three generators, three transformers, nine buses and nine transmission lines [19]. In order to highlight the effects of loop flow, the system was divided into three areas and a transfer of $10 \mathrm{MW}$ of active generating power from bus 2 to the load bus 8 , both belonging to the area 2 , is considered. The reference bus (bus 1, type $V \theta$ ) belongs to the reference area 1 . The buses 2 and 3 are the respective regulating buses (bus type $\mathrm{V}$ ) of the areas 2 and 3 .

Note that in Fig. 1, to attend the load increase of $10 \mathrm{MW}$ at bus 8 , the power flow divides between the two parallel existing paths between buses 7 and 8 . Note also that with area interchange controls, the power flow variations regarding to the base case in the three measurement points are equal. With the AIC, the power flow that will pass through the areas 1 and 3 will force their generations to correct their respective interchange deviations. Then, the power flow variation (1.020 MW) entering an area at a measurement point has to be the same as coming out of that area by another measurement point, once the net interchanges are kept constant at their schedule values of base case. Therefore, the loss variation caused by this loop flow, produces a generation increase of $0.006 \mathrm{MW}$ (equal to $0.010-0.004$ ) in area 1 , and a generation decrease of $0.038 \mathrm{MW}$ (equal to $0.008-0.046$ ) in area 3. The generation reduction of area 3 was due to the loss reduction $(-0.046 \mathrm{MW})$ with the counter flow (active power flow variation contrary to the base case direction) in the transmission line (TL) between the buses 6 and 9, which was larger than the increase of losses $(0.008 \mathrm{MW})$ due to the flow increase in TL between the buses 9 and 8 . So, the global effect in area 3 was losses reduction $(-0.038 \mathrm{MW})$. Note that it is common to find similar conditions in real power system [2-4,16-18]. 


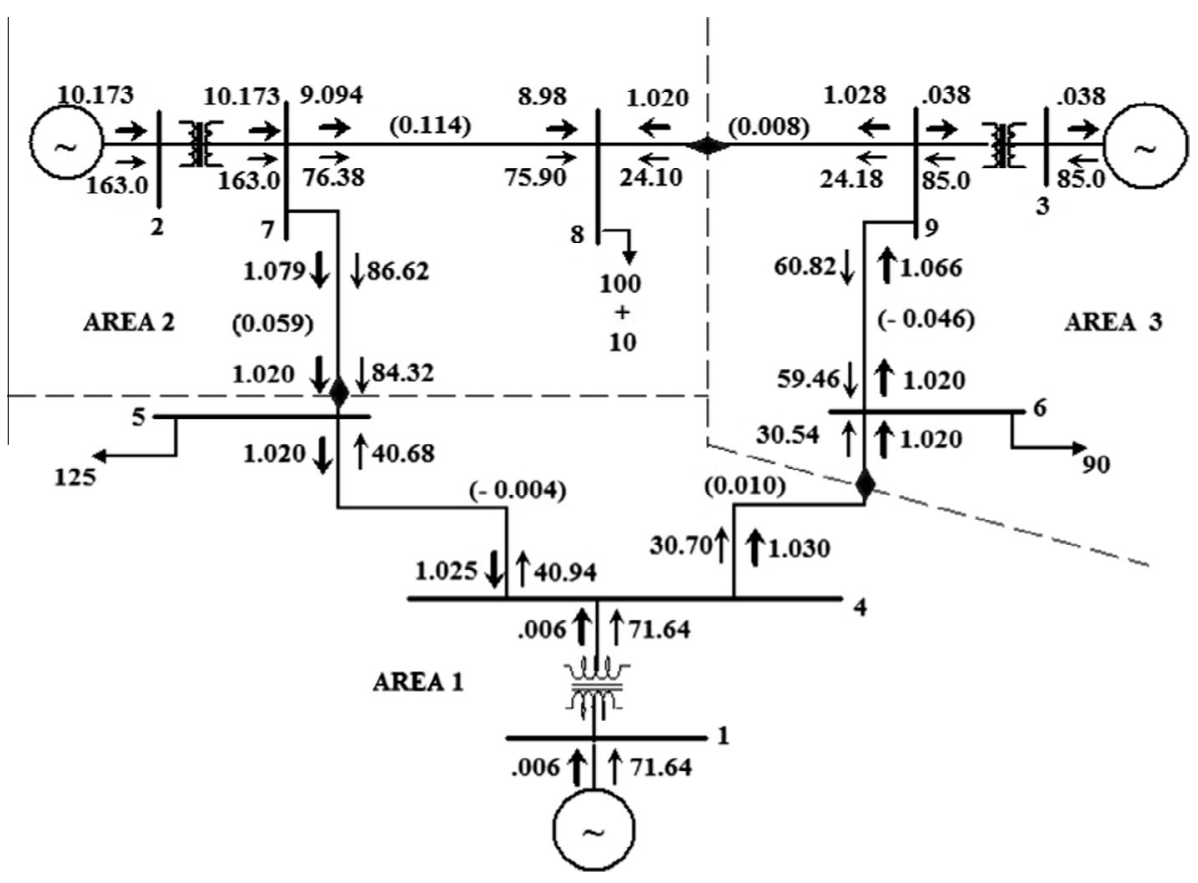

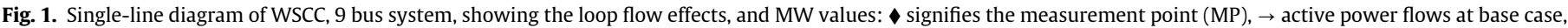
$\longrightarrow$ active power flow variations regarding to the base case, number in parentheses shows the loss variations regarding to the base case.

Performance of the proposed method to determine the power transfer capacity between two regions, at the same area

Fig. 2(a) shows the $P-V$ curves considering the active power load increase at the bus 8 , which is supplied by the generating bus 2 where both buses belong to area 2 . From the results, it can be concluded that the inclusion of the AIC control leads to a loading margin reduction of about $3.5 \%$.

As it is clear from Fig. 2(b), during all the load variation, the power flow variations at the three metering points are equal. Without the AIC, the whole system is treated as a single area and the bus 1 is considered as reference and therefore, it is responsible for maintaining the active power balance of the entire system, and supplying all loss variations of the system, as shown in Fig. 2(c) and (d). On the other hand, with the AIC, the loss variations of each area caused by the loop flow are supplied by their respective regulating buses to meet their scheduled interchanges, as illustrated in Fig. 2(e) and (f).

Performance of the proposed method to determine the power transfer capacity between areas

The results presented in Fig. 3 are obtained by considering the active power load increase at the bus 5 and unifying the areas 2 and 3 of Fig. 3 as a single area, namely as area 2. The active loads of bus 5 of the sink area 1 are increased proportionally to the base case and it is supplied by the regulating buses 2 and 3 of the source area 2 . The respective participation factors calculated from the base case by using the Eq. (4) are equal to $65.72 \%$ and $34.28 \%$. From the $V-\lambda$ curves of Fig. 3(a), it can be verified that there is a reduction of $8 \%$ on the active power transfer capacity, when compared to the case of without AIC.

Fig. 3(b) shows the total active generated power variations of regulating buses 2 and 3 (curve 1), the active loss variation within the area 2 (curve 2) and the power transfer (curve 3 ). Curve 4 is the resultant curve obtained by adding curves 2 and 3, point by point. This confirms that the total active power generated by the regulating buses of area 2 supplies its losses and the power transfer scheduled to meet the load increase at the bus 5 of area 1 , while the net interchanges are maintained according to the scheduling.

\section{Performance of the proposed CPF for the modified 118-bus IEEE test system}

As shown in Fig. 4, the system consisting of 118 busses, 186 branches and three separate areas is used to verify the performance of the proposed CPF. Bus 69, the slack bus (type $V \theta$ ), belongs to the area 2 , the reference area of the system. A power transfer from area 2 to area 3 is considered. The goal is to show the AIC influence on the power transfer capacity considering the inclusion of multiple AIC regulating buses per area. Two different power transfer scenarios are considered. In the first (scenario 1) a constant power factor is considered, and in the second (scenario 2) only the active loads are increased.

In the scenario 1 , the active and reactive loads of buses 78,79 , 82 and 83 of the sink area 3 are increased proportionally to the base case. The load increments must be supplied by the generating buses 46 and 49 of the source area 2, whose participation factors are calculated from the base case by using Eq. (4). The performances with and without the AIC are compared. In both cases, the bus 69 is the slack bus. When the AIC is considered, buses 6 and 69 are the respective regulating buses of area 1 and 2, while the generating buses 76 and 77 are the regulating buses of area 3. Their participating factors are equal to $66.66 \%$ and $33.33 \%$, respectively. The interchange is adjusted to allow for the power transfer between the areas. In this case, the interchange is increased in the source area 2, while it is decreased in the sink area 3. The interchange of area 1 is kept at its scheduled base case value.

In Fig. 5(a) the $V-\lambda$ curves of bus 82 for both cases are shown. It can be seen that the AIC has made an increase that is larger than $6 \%$ in the active power transfer capacity, when compared to without AIC (curve 1). Fig. 5(b) presents the summations of active power variations flowing into and out of area 1 . Curve 1 is the sum of active power flow variations at the metering points MP-1, MP-2 and MP-3, which is coincident with that flowing out of area 1 


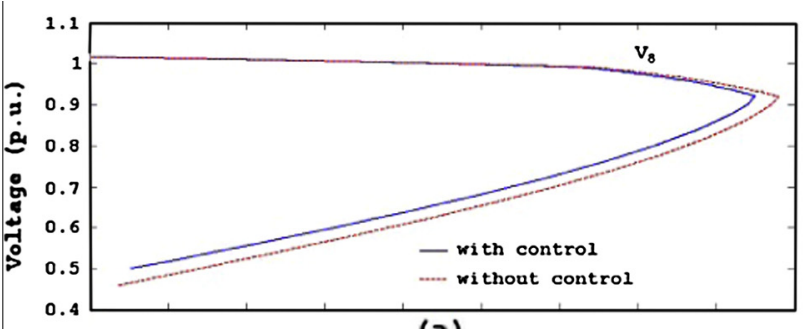

(a)

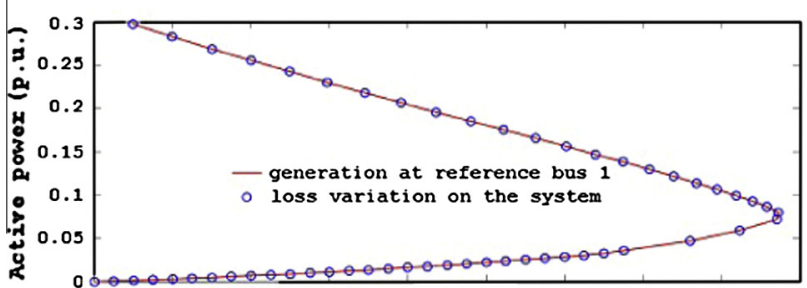

(c)

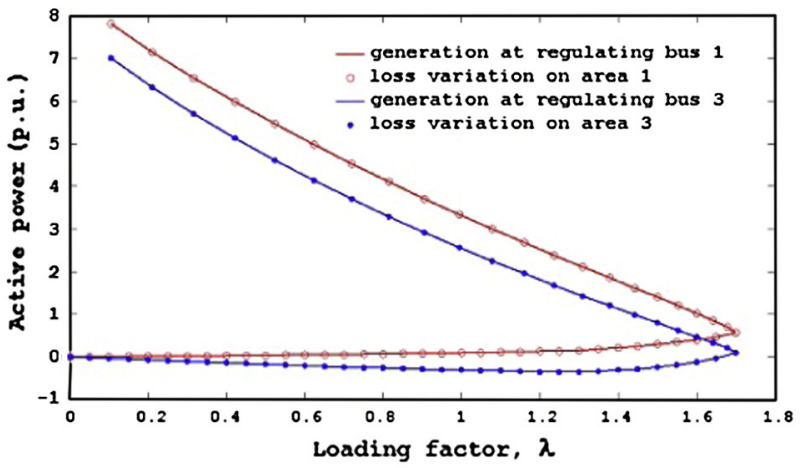

(e)
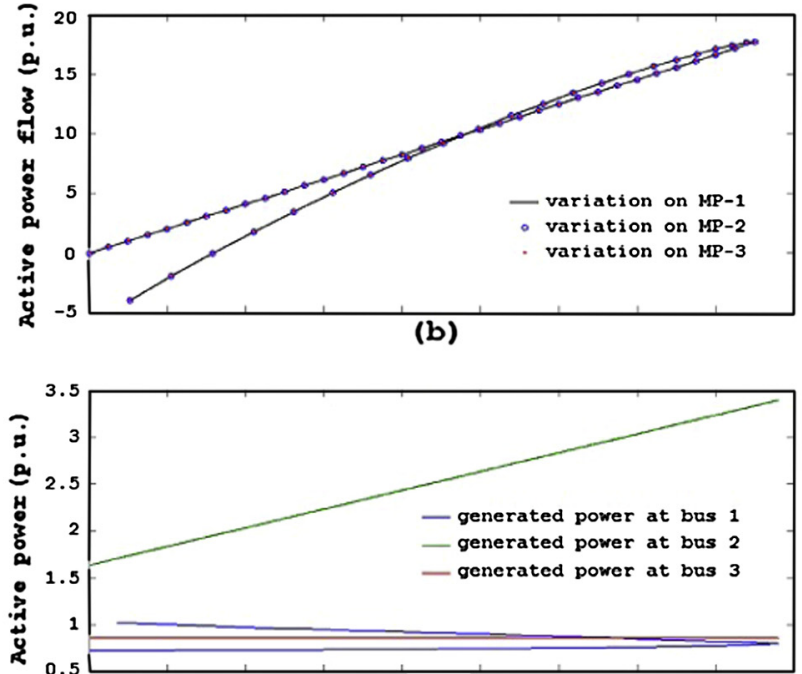

(d)

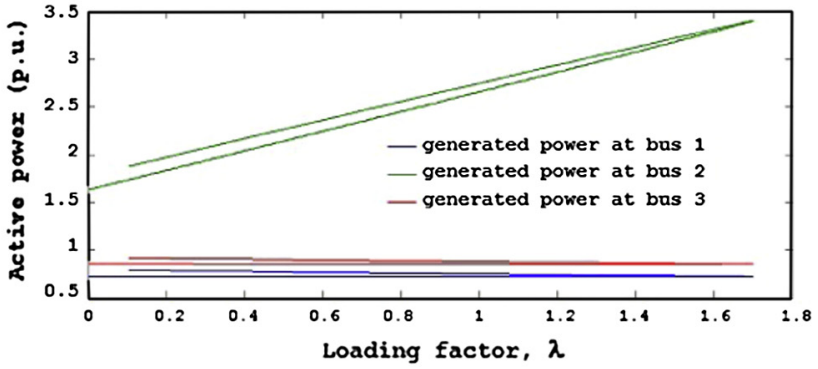

(f)

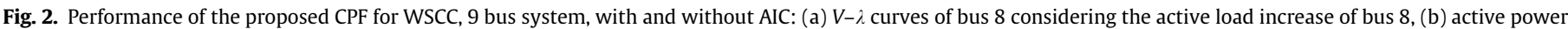

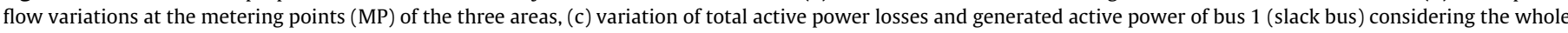

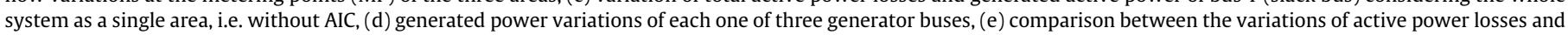
generated active power of regulating bus for areas 1 and 3, and (f)generated power variations of regulating buses of each area.

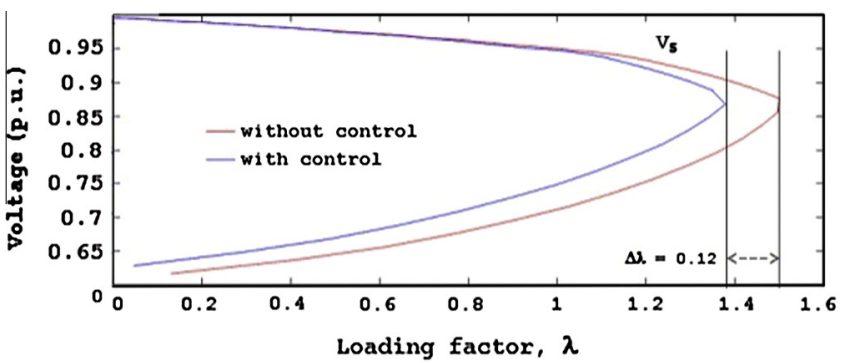

(a)

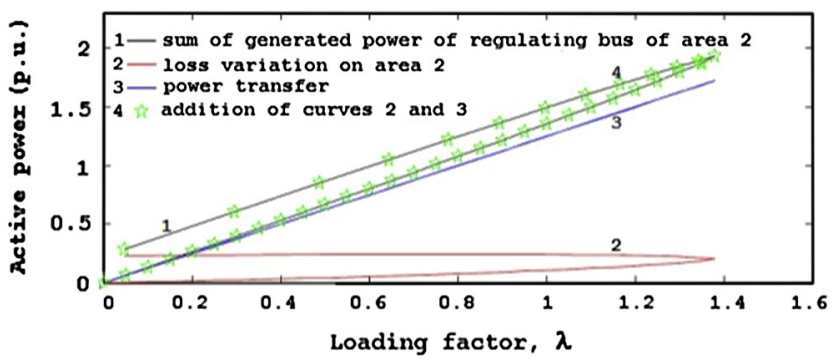

(b)

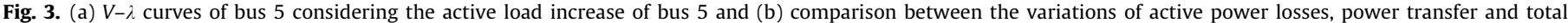
generated active power of regulating bus of area 2 .

through the MP-4 (curve 2), where the net interchange of area 1 is kept constant, as it can be seen in Fig. 5(c). Curves 1 and 2 in the Fig. 5(d) show the respective generated active power variations of buses 46 and 49 . It is also noted from this figure that the sum of the generated active power variations of these buses (curve 4 is the addition of curves 1 and 2 ) is always equal to the power transfer variation ( $\lambda$ ger $_{\mathbf{k}} \mathrm{Ptr}_{\mathbf{0}}$ ), which corresponds to the summation of the load increments of the sink area 3 supplied by the regulating buses ( 46 and 49) of the source area 2.

The loss variations of each area are supplied by their respective regulating buses to meet the scheduled interchanges. As shown in
Fig. 5(e), the regulating bus 6 supplies only the loss variations of area 1 , while the regulating bus 69 supplies the variation of the losses within the area 2. Meanwhile, as it can be seen from Fig. 5(f), the variations of active loss within the area 3 (curve 1) are shared by both regulating buses 76 and 77 (curves 2 and 3, respectively), according to their participation factors, which can be confirmed by adding curves 2 and 3 point by point, to give the curve 4 . Thus, ensuring that the net interchanges are maintained according to the scheduling, i.e. increasing in area 2 (curve 2 ), reducing in area 3 (curve 3 ), and keeping constant in area 1 , as shown in Fig. 5(c). It is worth mentioning in Fig. 5(g) that without 

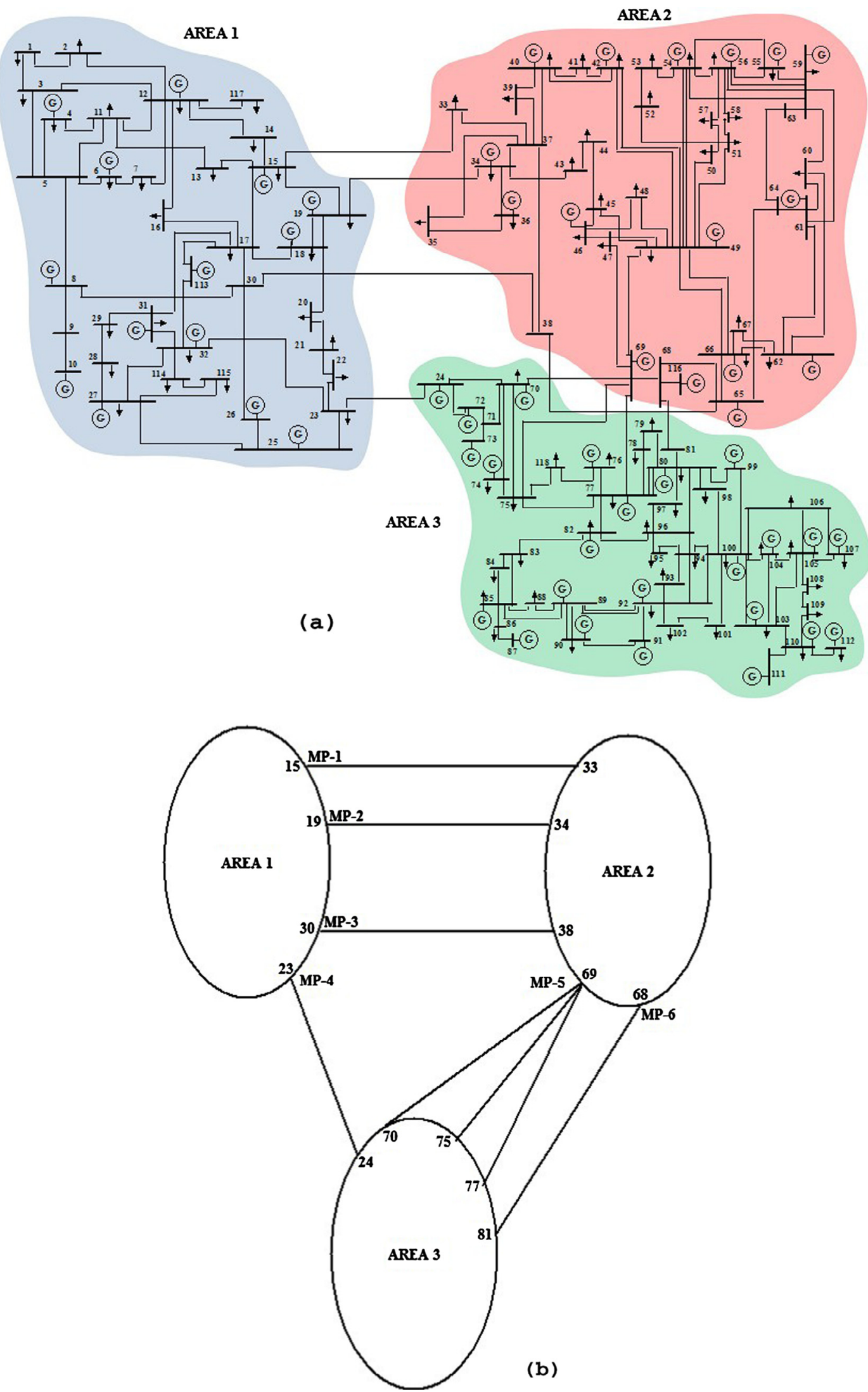

Fig. 4. IEEE 118-bus system: (a) single-line diagram arbitrary splits into three areas and (b) simplified three-area representation. 


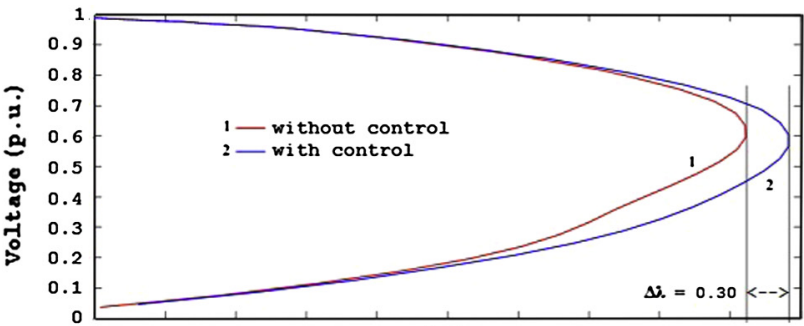

(a)

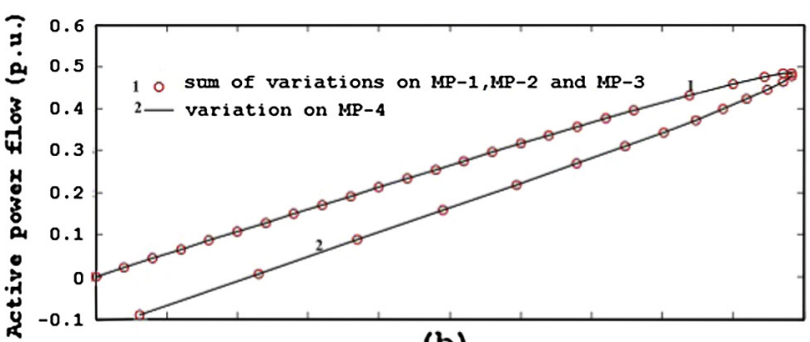

(b)

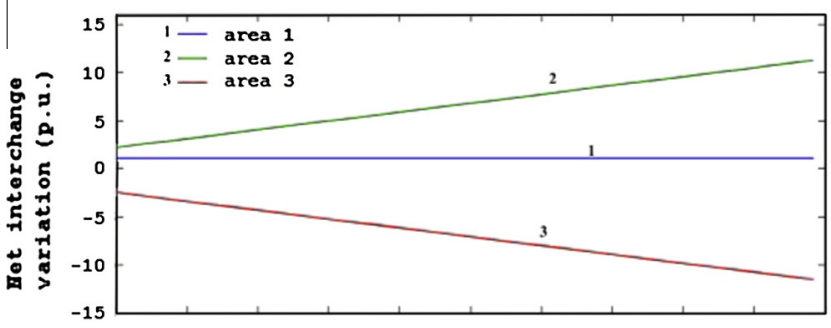

(c)

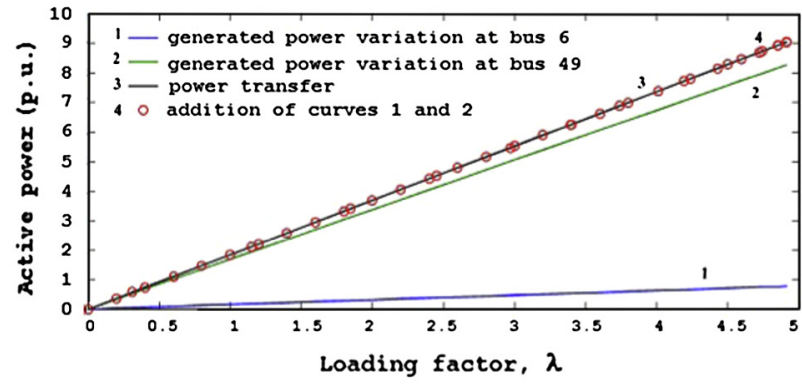

(d)

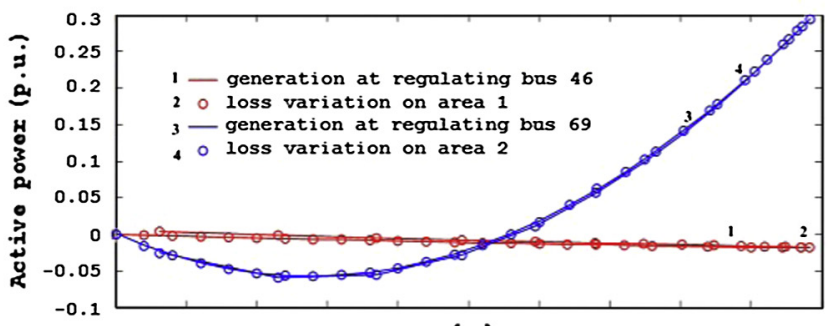

(e)

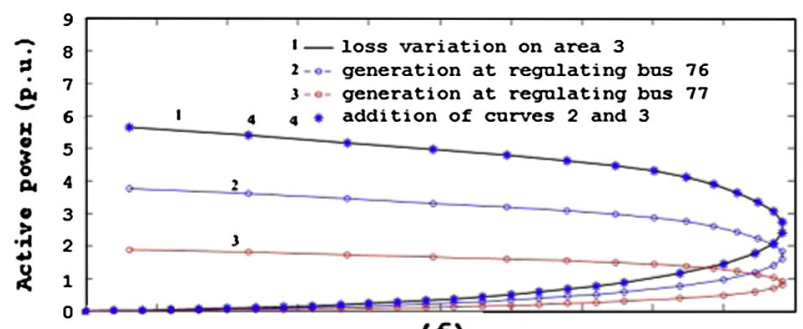

(f)

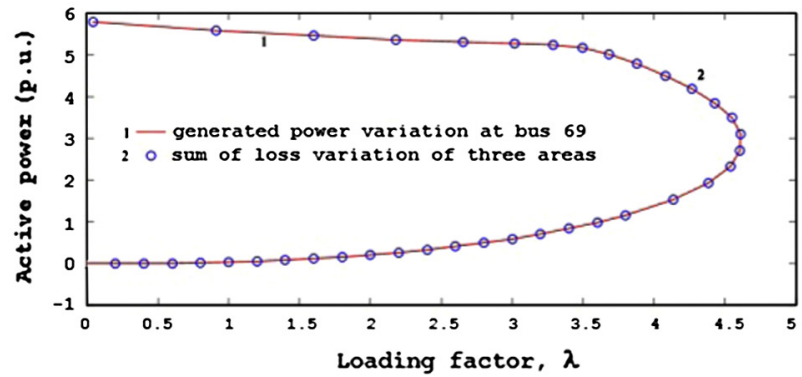

(g)

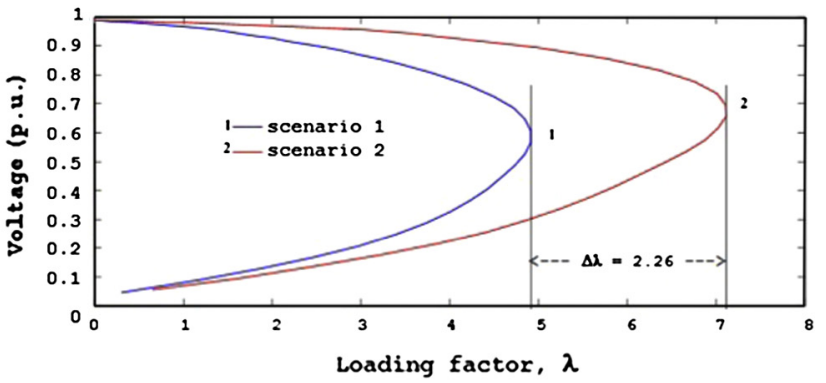

(h)

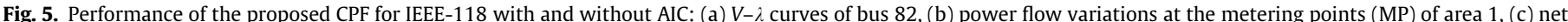

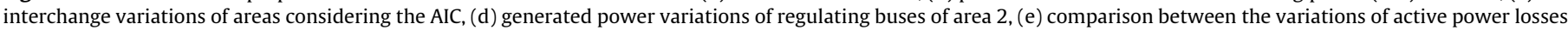

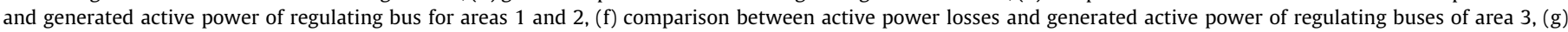

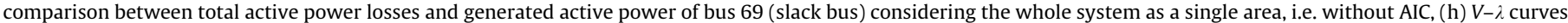
of bus 82 for two different load change scenarios: with a constant power factor and only with the active loads increase.

the AIC, the whole system is treated as a single area and the bus 69 is now considered as reference, i.e. it is responsible for maintaining the active power balance of the entire system, supplying all loss variations of the system.

Fig. 5(h) provides a comparison between scenario 1 , which considers the loads increase of the buses 78, 79, 82 and 83 with constant power factor, and scenario 2 , where only the active power loads of the aforementioned buses are increased. Comparing the $V-\lambda$ curves of bus 82 , it is verified a reduction of $31.9 \%$ in the power transfer capacity when constant power factor is considered. So, the simultaneous increase of reactive power is a considerable restriction on the ability to transfer power through the system.

\section{Performance of the proposed CPF for single branch outages}

An important issue for operating and planning studies of an interconnected area is related with the impact that external contingencies could cause on your own system, as well as those that internal contingencies could cause on neighboring systems $[20,21]$. Prior knowledge of potential problems enables members' utilities to be better prepared to identify and plan for critical contingencies and thus to prevent instability and a possible voltage collapse [20]. A commonly operation studies criteria recommended to mitigate the voltage-collapse requires that the utilities have at least a 5\% P-V margin, i.e. as measured from the MLP, under the worst single element contingency [21,22]. Additionally, it is also 


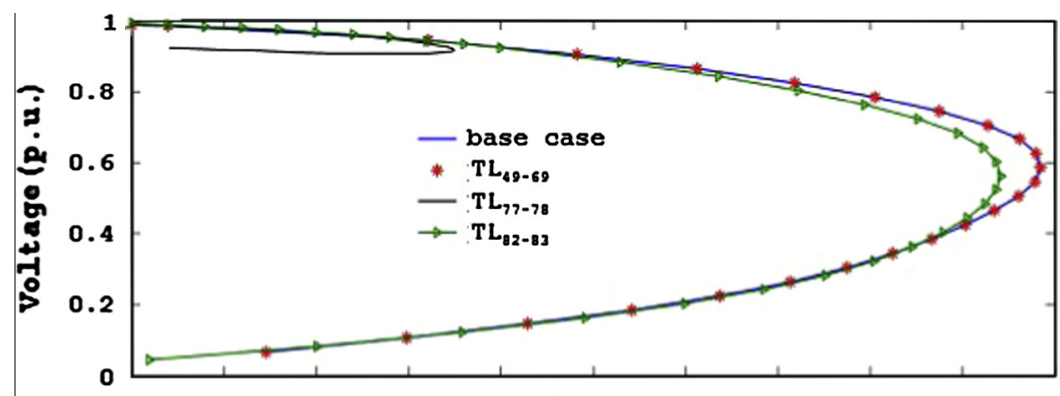

(a)

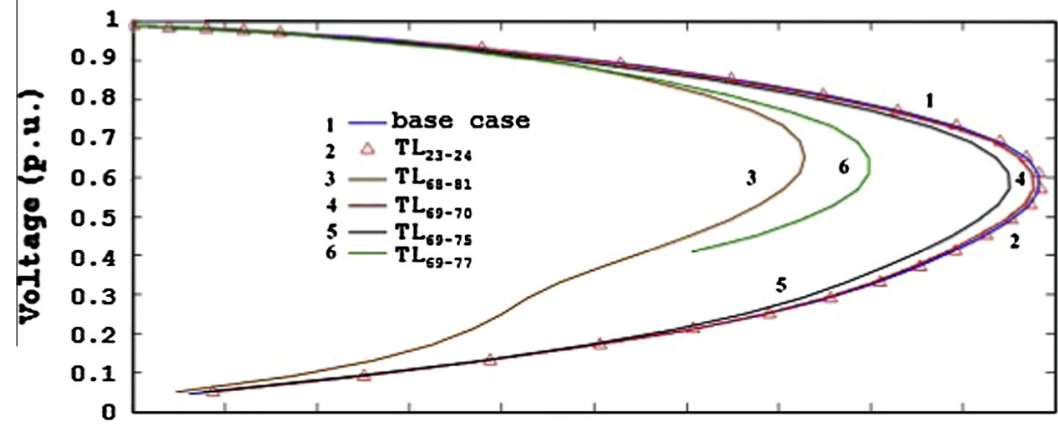

(b)

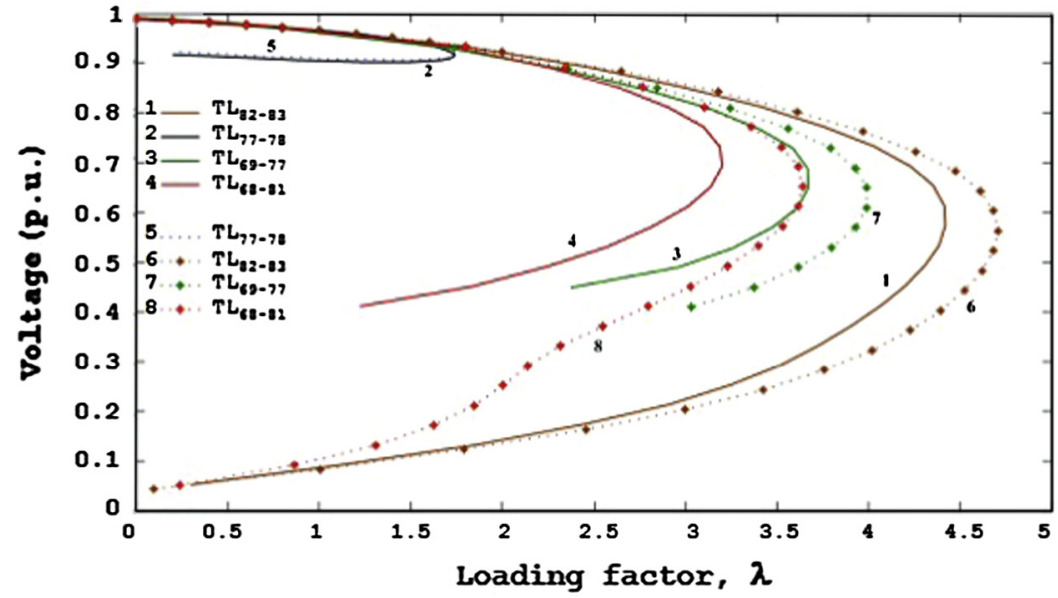

(c)

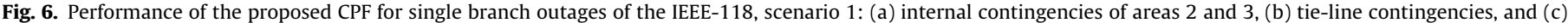
comparison between contingencies with and without AIC.

recommended that the pre and post-contingencies voltage must be within $0.95-1.05$ and $0.9-1.1$ p.u., respectively.

Fig. 6 presents the influence on the power transfer capacity of single branch outages of the scenario 1 of the IEEE-118. In Fig. 6(a) three internal transmission line (TL) contingencies are shown: in the area 2, the outages of the TL between the buses 49 and 69 and that between the buses 77 and 78, and in the area 3, the outage of the TL between the buses 82 and 83. In Fig. 6(b) the outages of the only tie-line that interconnects the areas 1 and 3, between the buses 23 and 24, and also each one of the four tie-lines that interconnects the areas 2 and 3 are shown.

Note that in from Fig. 6(a), when the system undergoes the internal contingency of TL between the buses 77 and 78, there is a considerable decrease in the active power transfer compared to the base case. This was expected since this LT is located in one of the most important path of the power transfer. On the other hand, the contingency of the TL located between the buses 82 and 83 causes only a slight decrease in active power transfer capacity, whereas the outage of TL located between the buses 49 and 69 practically provides no decrease in active power transfer capacity. Regarding to the contingencies of the tie-lines presented in Fig. 6(b), it can be noted that they generally often lead to a greater reduction in the active power transfer capacity compared to the base case. The most severe contingency corresponds to the tie-line located between the buses 68 and 81 . Note also that the outage of TL between buses 23 and 24 causes no decrease in active power transfer capacity, despite it is the only tie-line that interconnects the areas 1 and 3.

Fig. 6(c) shows a comparison with (dashed line) and without (solid line) considering the AIC for several TL contingencies. It can be seen from this figure that, the contingency of the tie-line between the buses 68 and 81 considering the AIC causes a difference up to $12 \%$ in the active power transfer compared to the same outage but without the control.

\section{Conclusion}

In this paper, the effects of the inclusion of the area interchange control on the active power transfer capacity were analyzed. It was 
shown the importance of including the equality constraint equations of net interchanges for assessing the maximum power transfer capacity, without violations of power system's static voltage stability. Furthermore, it shows that the proposed continuation power flow improves the calculation accuracy as well as provides more realistic results of a power system under different operating conditions. With the inclusion of the AIC, a difference of up to $12 \%$ in the power transfer capacity is verified. Also the greatest influence of the load increasing of the power system considering constant power factor on the active power transfer margin can be seen. It was observed that the most severe contingencies are generally the tie-lines and the transmission lines that are located in the main path of the power transfer. It was also shown that as a consequence of loop flows, an area that is not participating in the exchange of power has to cover the losses incurred by these flows and consequently, the power transfer capacity between two regions of its network may be restricted. Moreover, the AIC regulating buses will be increase or decrease their generations in order to provide the loss variations.

\section{References}

[1] WIKIPÉDIA. Electricity sector in Brazil; 2013. <http://en.wikipedia.org/wiki/ Electricity_sector_in_Brazil>.

[2] Congestion Management Process Working Group (CMPWG) - Midwest ISO and PJM. Investigation of loop flows across combined Midwest ISO and PJM footprint. Study report; 2007. p. 43. <http://www.miso-pjm.com/documents. aspx>.

[3] Congestion Management Process Working Group (CMPWG) - Midwest ISO and PJM. Investigation of loop flows across combined Midwest ISO and PJM footprint Phase II. Study report - final; 2008. p. 34. <http://www.misopjm.com/working-groups/joint-and-common-wg.aspx>.

[4] SINTEF Energy Research. Transit in European power market. Technical report; 2007. p. 102.

[5] Ajjarapu V. Computational techniques for voltage stability assessment and control. Power electronics and power systems series. New York: Springer; 2010.
[6] Garbelini E, Alves DA, Neto AB, Righeto E, da Silva LCP, Castro CA. An efficient geometric parameterization technique for the continuation power flow. Electr Power Syst Res 2007;77(1):71-82.

[7] Bonini Neto A, Alves DA. Improved geometric parameterization techniques for continuation power flow. IET Gener, Transm Distrib 2010;4(12):1349-59.

[8] Yang X, Zhou X, Ma Y, Du Z. Asymptotic numerical method for continuation power flow. Electr Power Energy Syst 2012;43:670-9.

[9] Razmi H, Shayanfar HA, Teshnehlab M. Steady state voltage stability with AVR voltage constraints. Electr Power Energy Syst 2012;43:650-6599.

[10] Monticelli AJ. Power flow in electric networks. São Paulo, Brazil: Edgard Blucher; 1983 (In Portuguese).

[11] Dos Santos MJ, Resende JL, Filho JAP, Oliveira EJ, Silva Jr IC. A new approach for area interchange control modeling. IEEE Trans Power Syst 2004;15:1093-7.

[12] Ibsais A, Ajjarapu V. Voltage stability-limited interchange flow. Electr Power Syst Res 1996;38:91-5.

[13] Echavarren FM, Lobato E, Rouco L, Gómez T. Formulation, computation and improvement of steady state security margins in power systems Part I: Theoretical framework. Int J Electr Power Energy Syst 2011;33(2):340-6.

[14] Acharjee P. Identification of maximum loadability limit and weak buses using security constraint genetic algorithm. Electr Power Energy Syst 2012;36: 40-50.

[15] Mallick S, Acharjee P, Ghoshal SP, Thakur SS. Determination of maximum load margin using fuzzy logic. Electr Power Energy Syst 2013;52:231-46.

[16] Miller JM, Balmat BM, Morris KN, Malinowski JH, Pasternack BM, Eilts LE. Operating problems with parallel flows. IEEE Trans. Power Syst 1991;6(3): 1024-34 [IEEE committee report].

[17] Choo CY, Nair NC, Chakrabarti B. Impacts of loop flow on electricity market design. In: International conference on power system technology - PowerCon; 2006. p. 1-8.

[18] Abdel-Rahman K, Phadke A, Liu Y. A tracing load flow program for total transfer capability calculations, In: IEEE power engineering society summer meeting; 2002. p. 1439-43.

[19] Anderson PM, Fouad AA. Power system control and stability. IEEE Press; 1994

[20] The North American Electric Reliability Corporation (NERC). Summer assessments. 2012 Summer reliability assessment; 2012. p. 195. <http:// www.nerc.com/pa/RAPA/ra/ReliabilityAssessmentsDL/>.

[21] The North American Electric Reliability Corporation (NERC). Methods and assumptions. 2012 Long-term assessment methods and assumptions; 2012. p. 79. <http://www.nerc.com/pa/RAPA/ra/ReliabilityAssessmentsDL/>.

[22] Reactive Power Reserve Work Group. Voltage stability criteria, undervoltage load shedding strategy, and reactive power reserve monitoring methodology; 1998. p. 154. <http://www.wecc.biz/library/DocumentationCategorizationFiles/ Guidelines> (File name: Voltage Stability Criteria - Guideline, Date adopted/ approved April 2010). 